\title{
Enfermedad de Gaucher: una enfermedad multisistémica
}

\author{
Gaucher Disease: a multisystemic disorder
}

\author{
Alberto Rivera Gallego, Rut Lorenzo Castro, Iria Villaverde Álvarez \\ Unidad de Enfermedades Minoritarias Sistémicas. Servicio de Medicina Interna. Complexo Hospitalario de Vigo
}

\section{RESUMEN}

La enfermedad de Gaucher es un trastorno autosómico recesivo raro debido a la ausencia de la enzima glucocerebrosidasa, produciéndose acumulación de glucocerebrósidos en el sistema retículo endotelial. Se manifiesta por hepatoesplenomegalia, alteraciones hemáticas, neurológicas y óseas. Presentamos el caso de una paciente de 19 años de edad que ingresa por dolor abdominal generalizado, cansancio y debilidad, encontrándose hallazgos concordantes con una afectación infiltrativa en vertebras y ambas diáfisis femorales junto con necrosis avascular de ambas cabezas femorales. Se evidenciaron niveles de $\beta$ glucosidasa disminuidos, y en el estudio genético se encontró la mutación p.Asn409Ser en homocigosis. La terapia de reemplazo enzimático logró normalización de cifras hematológicas al cabo de 6 meses y de tamaño de bazo e hígado al cabo de 1 año.

Palabras clave: enfermedad de Gaucher, Glucocerebrosidasa, Enfermedad de Depósito Lisosomal, esplenomegalia, trombocitopenia, Terapia de Reemplazo Enzimático (TRE).

\section{INTRODUCCIÓN}

La enfermedad de Gaucher (EG), transmitida de forma autosómica recesiva, es el mas común de los trastornos de depósito lisosomal. Está causada por mutaciones en el gen GBA1, cuyo resultado es una deficiencia en la actividad de la glucocerebrosidasa (GCasa) acumulándose su sustrato, la glucosilceramida en los lisosomas de los macrófagos (células de Gaucher), fundamentalmente en el bazo, hígado y médula ósea. Como consecuencia resaltar la presencia de citopenias, organomegalias y alteraciones óseas. El diagnóstico se basa en la medición de la actividad enzimática y el estudio genético. Los tratamientos aprobados hasta la fecha son el reemplazamiento enzimático (TRE) 0 la reducción de sustrato (TRS) 1 .

Se reporta el caso de una paciente con EG tipo 1 que se presenta con esplenomegalia masiva y citopenias.

\section{CASO CLÍNICO}

Se trata de una paciente de 19 años que ingresa por dolor abdominal generalizado, cansancio y debilidad. Raza caucásica y padres sin cosanguineidad. No refería antecedentes familiares de interés, incluidos sus padres y un hermano.

El embarazo fue normal y a término. Diagnosticada de una Enfermedad de Perthes bilateral a los 9 años que se manejó mediante tratamiento conservador, por el resto el desarrollo psicomotor fue normal. No refería historia de hemorragia anormal, ni tampoco fiebre, sudores nocturnos, pérdida de peso o dolores óseos.

En el examen físico destacaba palidez cutánea y hemorragia conjuntival en cuadrante inferior de ojo derecho. Tenía

\begin{abstract}
Gaucher disease is a rare autosomal recessive disorder due to the absence of the enzyme glucocerebrosidase, causing accumulation of glucocerebrosides in the reticulum endothelial system. It is manifested by hematologic abnormalities, hepatosplenomegaly, and neurological and bone manifestations. We present the case of a 19-year-old patient who was admitted due to generalized abdominal pain, fatigue and weakness, along with infiltrative involvement in vertebrae and both femoral diaphyses together with avascular necrosis of both femoral heads. Decreased levels of $\beta$-glucosidase were evidenced, and the genetic study found the p.Asn409Ser mutation in homozygosity. Enzyme replacement therapy achieved normalization of hematological figures after 6 months and of spleen and liver size after 1 year.
\end{abstract}

Keywords: Gaucher disease (GD), lysosomal storage disorder, glucocerebrosidase, splenomegaly; thrombocytopenia, Enzyme Replacement Therapy (ERT).

esplenomegalia de 5 traveses por debajo de reborde costal no dolorosa y una hepatomegalia leve de 1 través que era lisa y sin reflujo hepato-yugular. No adenomegalias. El resto de la exploración física, incluyendo neurológica, fue normal. Los análisis de laboratorio revelaron citopenias (hemoglobina $9.2 \mathrm{~g} / \mathrm{dl}$, glóbulos blancos $1.94 \mathrm{mil} / \mathrm{mm}^{3}$ y plaquetas $\left.79000 / \mathrm{mm}^{3}\right)$. El resto de la analítica fue normal incluyendo proteinograma e inmunofijación, autoinmunidad, cultivos de sangre y orina, serologías y Mantoux. La ecografía abdominal fue informada como bazo de diámetro longitudinal de $20 \mathrm{~cm}$ e hígado aumentado de tamaño, ambos con ecoestructura normal, sin evidenciarse otras alteraciones. En la tomografía computerizada (TC) toraco-abdominal se objetivó hepatomegalia de $19 \mathrm{~cm}$ y esplenomegalia de $20.5 \mathrm{~cm}$ sin otras alteraciones relevantes. Se realizó biopsia de médula ósea que reveló acúmulos nodulares de forma difusa de células de aspecto histiocitario, con amplios eosinófilos fibrilares, PAS positivos y con depósitos focales de hemosiderina en un fondo de células normales de linaje eritroide, mieloide y megacariocítico. En la resonancia magnética (RM) ósea se encontraron hallazgos concordantes con una afectación infiltrativa en vertebras y ambas diáfisis femorales, además de imágenes sugestivas de infartos óseos a nivel de región intertrocantérea y cuello de fémur izquierdo, junto con necrosis avascular de ambas cabezas femorales (figura 1). Se evidenciaron niveles de GCasa disminuidos y en el estudio genético se encontró la mutación p.Asn409Ser en homocigosis. El diagnóstico final fue EG tipo 1. Se inició TRE presentando normalización de cifras hematológicas al cabo de 6 meses y de tamaño de bazo e hígado al cabo de 1 año. 


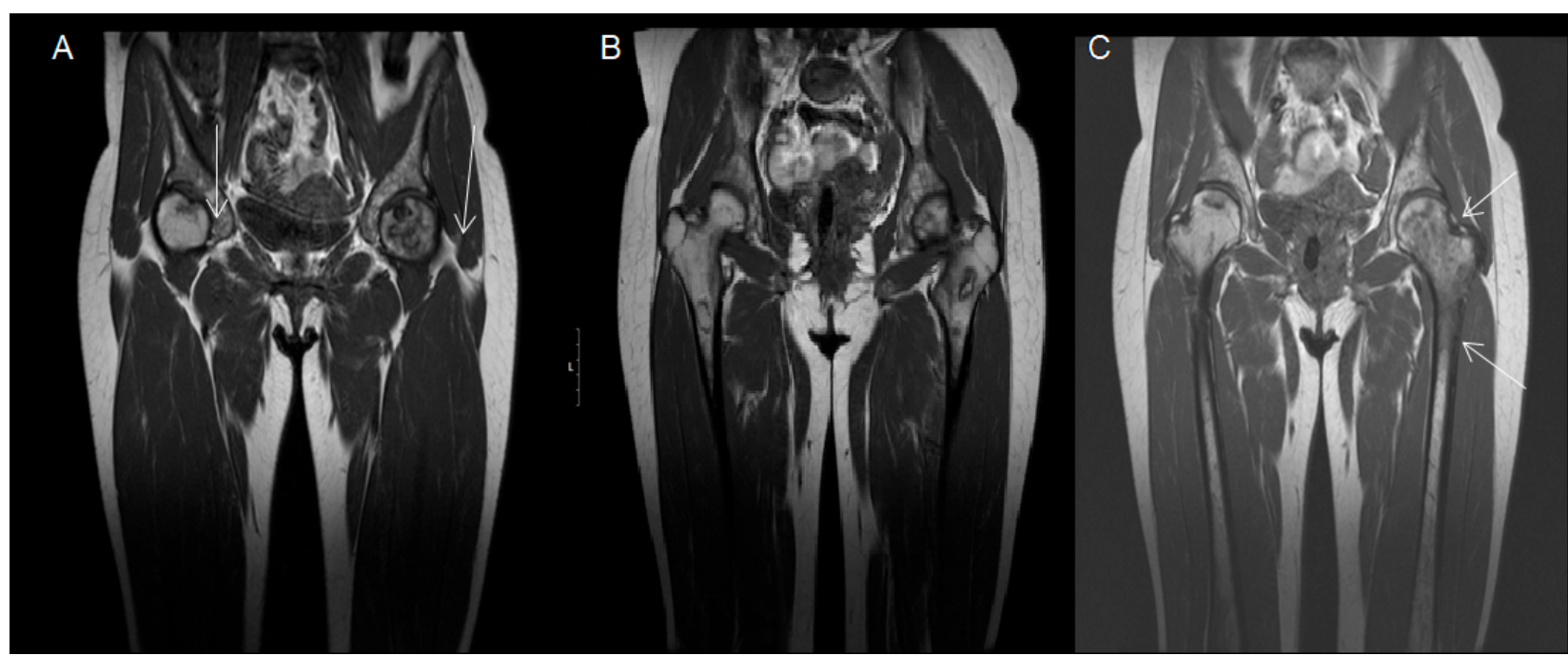

\section{DISCUSIÓN}

La prevalencia aproximada de la EG al nacimiento se calcula 1/40.000 (en la población general) a 1/850 (en judíos Askenazi) $)^{2}$. Actualmente, se han descrito más de 300 mutaciones responsables en el gen de la glucocerebrosidasa humana (cromosoma 1q22), algunos muy comunes, como la mutación p.Asn409Ser y p.Leu483Pro ${ }^{3}$. Aunque existen correlaciones genotipo-fenotipo, en un individuo concreto el genotipo no predice el pronóstico².

Las mutaciones en el gen GBA1 conducen a una acumulación de glucosilceramida en los lisosomas de las células del sistema retículo-endotelial, especialmente en los macrófagos, provocando su transformación en las denominadas células Gaucher (CG). Estas formas celulares son un fenotipo de macrófago activado con propiedades antiinflamatorias, inmunomodulatorias y de reparación de tejido, con un perfil de enzimas lisosomales (incluyendo la chitotriosidasa) y citoquinas y quimioquinas (incluyendo IL-1 beta, IL-6, TNFalpha, IL-10, IL-18 y CCL18) que le confiere un carácter sistémico a la EG ${ }^{4}$. Las CG infiltran el tejido hepático, esplénico, médula ósea y otros órganos ocasionando organomegalia y fragilidad ósea (incremento de la reabsorción trabecular, aparición de áreas de adelgazamiento cortical y áreas líticas óseas). Esta expansión ósea promueve la compresión extrínseca de los vasos intraóseos que, combinado con una modificación del microambiente espinal y anomalías de los globulos rojos, dan lugar a una isquemia ósea causando infartos y osteonecrosis.

La presentación de la EG es muy heterogénea, variando desde la forma asintomática a la letal. Aunque clásicamente se diferencian tres fenotipos y una forma fetal, se considera un continuo entre estos diferentes tipos, basado en la presencia e intensidad de las manifestaciones neurológicas ${ }^{5,6}$.

La EG Tipo 1 es la más común (95\% de los casos), con una mediana de edad de aparición de los primeros síntomas de 15 años (0-77 años) y una mediana de edad al diagnóstico de 22 años (0-84 años) $)^{7}$. Su presentación clínica varía desde formas asintomáticas hasta formas muy severas. La afectación de cada órgano es muy variable y su evolución clínica son muy variables, incluso entre los miembros de una misma familia.

La esplenomegalia se presenta en más del $90 \%$ de los pacientes. Es el principal mecanismo de las citopenias dominada por la trombopenia. Se puede añadir el infarto esplénico, aunque su rotura es excepcional. Hasta un $60-80 \%$ de los casos se asocia con hepatomegalia? Se pueden encontrar lesiones focales en bazo o hígado, llamadas gaucheromas. El daño óseo se observa hasta en el 80\% de los casos (infarto óseo, osteonecrosis aséptica, osteoporosis, retraso del crecimiento, deformación en matraz de Erlenmeyer). Las ubicaciones preferentes son la columna vertebral, la pelvis y las extremidades inferiores. Estos eventos pueden ser asintomáticos 0 paucisintomáticos, siendo necesario una investigación sistemática mediante pruebas de imagen ${ }^{8}$. La astenia es común en estos pacientes. De manera excepcional, está descrita la afectación cutánea (pigmentación amarillo-marrón en la cara y las tibias), pulmonar (neumonía intersticial, hipertensión pulmonar), neuropatía periférica, afectación cardíaca (pericarditis constrictiva), digestiva (enteropatía exudativa, cólicos), ocular (manchas blancas, vasculitis retiniana) y renal (síndrome nefrótico, insuficiencia renal). En mujeres es más común el retraso en la menarquia, la menorragia y complicaciones relacionadas con el embarazo ${ }^{9}$.

La EG Tipo 2 es una forma más rara, que generalmente comienza a los 3-6 meses de edad con hepatosplenomegalia y deterioro neurológico. Son muy sugestivos de la enfermedad la combinación de rigidez del cuello y opistótonos, signos bulbares (incluyendo trastornos de la deglución) y parálisis horizontal de la mirada ${ }^{4}$. El curso es desfavorable y la muerte ocurre antes del tercer año de vida principalmente debido a una afectación pulmonar infiltrativa o por apnea central.

La EG tipo 3, representa menos del 5\% de casos. La expre- 
Tabla 1. Objetivos a corto y largo plazo (modificado de Biegstraaten, M, et al 2018).

\begin{tabular}{|c|c|c|}
\hline & Corto plazo & Largo plazo \\
\hline Anemia & $\begin{array}{l}\text { Eliminar dependencia de transfusiones de sangre } \\
\text { Aumentar los niveles de HGB en un plazo de } 12 \text { a } 24 \text { meses a valores normales para } \\
\text { edad y sexo }\end{array}$ & Mantener niveles normales de HGB \\
\hline $\begin{array}{l}\text { Tendencia al } \\
\text { sangrado }\end{array}$ & $\begin{array}{l}\text { Aumentar el recuento plaquetario lo suficiente como para prevenir el sangrado } \\
\text { quirúrgico, obstétrico y espontáneo } \\
\text { En pacientes con esplenectomía - normalización del recuento plaquetario al } 1 \text { año de } \\
\text { tratamiento } \\
\text { En pacientes con bazo intacto: lograr el recuento plaquetario de } \geq 100.000 / \mathrm{mm} 3 \text { al } \\
\text { tercer } 3 \text { año de tratamiento }\end{array}$ & $\begin{array}{l}\text { Mantener el recuento plaquetario } \geq \\
100.000 / \mathrm{mm} 3\end{array}$ \\
\hline Hueso & $\begin{array}{l}\text { Disminuir el dolor óseo no relacionado con enfermedad ósea irreversible a los } 1 \text { ó } 2 \\
\text { años } \\
\text { Disminuir la afectación de la médula ósea, medida por un score } \\
\text { Aumentar la densidad mineral ósea (DM0) a los } 2 \text { años en adultos en pacientes con } \\
\text { una puntuación T inferior a -2,5 al diagnóstico } \\
\text { Mantener una movilidad normal o, si está afectada al diagnóstico, mejorar la } \\
\text { movilidad } \\
\text { Normalizar el crecimiento dentro de los } 2 \text { años posteriores al inicio del tratamiento }\end{array}$ & $\begin{array}{l}\text { Prevenir las complicaciones óseas } \\
\text { Prevenir la osteopenia/osteoporosis } \\
\text { (medida por DEXA) } \\
\text { Prevenir el uso crónico de analgésicos } \\
\text { Alcanzar una masa esquelética } \\
\text { máxima normal o ideal en niños }\end{array}$ \\
\hline Vísceras & $\begin{array}{l}\text { Evitar la esplenectomía } \\
\text { Alivio de síntomas secundarios a la esplenomegalia } \\
\text { Eliminar el hiperesplenismo } \\
\text { Disminución del volumen esplénico entre <2 a } 8 \text { veces dependiendo del volumen } \\
\text { inicial entre } 1-2 \text { años } \\
\text { Reducción del volumen hepático a 1-1.5 veces lo normal dentro de los } 1-2 \text { años }\end{array}$ & $\begin{array}{l}\text { Mantener el volumen del bazo de }<2 \\
\text { a } 8 \text { veces lo normal } \\
\text { Mantener un volumen normal o cerca } \\
\text { de lo normal del hígado } \\
\text { Prevenir la fibrosis hepática, la cirrosis } \\
\text { y la hipertensión portal }\end{array}$ \\
\hline Bienestar & $\begin{array}{l}\text { Mejorar las puntuaciones iniciales de un score validado de calidad de vida en un } \\
\text { plazo de } 2 \text { a } 3 \text { años, dependiendo de la carga de enfermedad } \\
\text { Reducir la fatiga (no relacionada con la anemia) medida por un score validado } \\
\text { Mejorar o restaurar la función física para llevar a cabo actividades normales }\end{array}$ & $\begin{array}{l}\text { Mantener una buena calidad de vida } \\
\text { medida por un score validado } \\
\text { Mantener una participación normal en } \\
\text { actividades } \\
\text { Minimizar las cargas psicosociales del } \\
\text { tratamiento de por vida } \\
\text { Lograr un inicio normal de la pubertad } \\
\text { Normalizar la esperanza de vida }\end{array}$ \\
\hline $\begin{array}{l}\text { Complicaciones } \\
\text { pulmonares }\end{array}$ & & $\begin{array}{l}\text { Prevenir o mejorar la enfermedad } \\
\text { pulmonar, la hipertensión pulmonar y } \\
\text { el síndrome hepatopulmonar }\end{array}$ \\
\hline $\begin{array}{l}\text { Embarazo y } \\
\text { parto }\end{array}$ & & $\begin{array}{l}\text { Prevenir las complicaciones } \\
\text { relacionadas con la GD durante el } \\
\text { embarazo y el parto }\end{array}$ \\
\hline
\end{tabular}


sión clínica es muy heterogénea, con un inicio, en general, antes de los 2 años. Además de los trastornos del tipo 1 se añaden signos neurológicos (oftalmoplegia horizontal supranuclear, síndrome cerebeloso y encefalopatía progresiva asociada a una epilepsia mioclónica, mioclonia no epiléptica, espasticidad, trastornos del comportamiento 0 incluso demencia). La clínica neurológica puede ocurrir varios años antes de otras manifestaciones clínicas ${ }^{4}$

Entre las anomalías de laboratorio en la EG se encuentran la trombopenia es frecuente ( $90 \%$ de los casos) y de diversos grados $(<60 \mathrm{G} / \mathrm{L} \text { en el } 26 \% \text { de los casos) })^{10}$. La trombopatía, frecuentemente asociada con esta trombopenia puede agravar la sintomatología hemorrágica en estos pacientes. La anemia suele ser moderada (56\% casos) y puede haber leucopenia, aunque no frecuente.

Es común la elevación de la ferritina (85\%), mientras que el hierro y el índice de saturación de transferrina son normales. Existe una elevación de enzimas lisosomales en plasma, incluyendo la enzima convertidora de angiotensina, la fosfatasa ácida resistente a tartrato y la hexosaminidasa. La hipergammaglobulinemia policlonal común (25-91\%), pudiendo presentarse uno o varios picos monoclonales (1-35\%), generalmente clasificada como de significado incierto ${ }^{11}$.

Actualmente, los biomarcadores más útiles en el seguimiento de pacientes con EG son la quitotriosidasa, CCL18 y la glucosilesfingosina. La quitotriosidasa, producida por las células de Gaucher se puede utilizar como marcador pronóstico y de eficacia; sin embargo, un 6\% de la población no la produce y alrededor del $30 \%$ produce poco. El CCL18 se produce en los macrófagos y células dendríticas, presenta una cinética similar a la quitotriosidasa, asociándose niveles altos con un pronóstico más grave. La sensibilidad y especificidad de la glucosilesfingosina (Lyso GL1) parecen ser superiores, correlacionándose con la hepatomegalia, esplenomegalia, esplenectomía y el tipo de tratamiento ${ }^{12}$.

La ecografía abdominal, por su disponibilidad, sigue siendo ampliamente utilizada como primera línea. La RM abdominal es el examen de elección para evaluar las dimensionesy morfología de bazo e hígado ${ }^{13}$ así como el daño óseo. Cuantifica el grado de infiltración ósea, evalúa el alcance de las lesiones, su temporalidad y la reducción del grado de infiltración durante la enfermedad ${ }^{14}$. Las radiografías óseas son útiles para el diagnóstico y el seguimiento de lesiones sintomáticas, la osteonecrosis y la valoración de fracturas, artrosis y osteosíntesis. La densitometría ósea puede detectar osteopenia o una osoteoporosis, común en EG ${ }^{8}$. Otros exámenes útiles son la TC torácica, pruebas de función respiratoria, el ecocardiograma, el electroencefalograma y la electrooculografía en casos de EG tipo 2/3.

Entre las complicaciones asociadas a la EG se encuentran:

- Parkinson. En pacientes con EG de tipo 1 el riesgo de Enfermedad de Parkinson (EP) aumenta de 6 a 17 veces en comparación con la población general, siendo de inicio más precoz. Al mismo tiempo, existe una fuerte asociación entre las mutaciones del gen GBA y EP, especialmente la mutación p.Leu483Pro 4 .

- Neoplasias. Parece existir un incremento en la incidencia de linfomas, hepatocarcinoma, melanoma y cáncer de páncreas 4 .

- Mieloma múltiple. Su incidencia está aumentada, con un riesgo relativo estimado de 37.5-51.5 comparado con la población general ${ }^{6}$.

\section{Diagnóstico}

La EG debe sospecharse en individuos con la combinación de afectación del sistema nervioso central, óseo, hematológico y otros hallazgos clínicos. El diagnóstico se establece mediante la demostración de un déficit (menor al 15\%) de GCasa en leucocitos de sangre periférica (u otras células nucleadas) o la identificación de variantes patógenas en GBA en las pruebas genéticas ${ }^{3}$.

Se ha desarrollado un test ultramicrofluorométrico para el diagnóstico de EG a partir de sangre seca en papel filtrante que facilita el diagnóstico ${ }^{4}$.

\section{Tratamiento}

El seguimiento de los pacientes con tratamiento lo establece un documento de consenso, publicado por un grupo de trabajo europeo, con los objetivos a corto y largo plazo ${ }^{15}$ (Tabla 1). Asimismo, existe un nuevo sistema de puntuación de gravedad de la enfermedad (DS3), utilizado para valorar los resultados de salud a largo plazo de las personas con EG1 ${ }^{16}$.

Existen dos opciones aprobadas para el tratamiento en la EG: tratamiento de reemplazamiento enzimático (TRE) y de reducción de sustrato (TRS). Otros tratamientos (las chaperonas y la terapia génica) están en investigación $n^{6,17}$.

\section{Tratamiento de Reemplazamiento Enzimático (TRE) ${ }^{17}$.}

Existen tres preparaciones enzimáticas: imiglucerasa (Cerezyme ${ }^{\circledR}$ ), velalglucerasa alfa (VPRIV ${ }^{\circledR}$ ) y taliglucerasa alfa $\left(\right.$ Elelyso $^{\circledR}$ ) (esta última no aprobada en Europa). Se ha demostrado que el TRE es eficaz en revertir las alteraciones de la afectación hematológica y visceral (hígado/bazo). Las manifestaciones neurológicas así como las somáticas crónicas parecen responder poco al tratamiento, pudiendo persistir la trombocitopenia en individuos con esplenomegalia residual y/o la presencia de nódulos esplénicos. En la mayoría de los individuos, el tratamiento se inicia con una dosis entre 15-60 unidades $/ \mathrm{kg}$ de peso corporal administrado por vía intravenosa cada dos semanas. Esta dosis se incrementa o disminuye en función de la consecución de objetivos? ${ }^{7}$. El TRE es bien tolerado. Aproximadamente 10\%-15\% y un 1\% de los individuos desarrollan anticuerpos contra la imiglucerasa y velaglucerasa respectivamente, permaneciendo asintomáticos en la mayoría de los casos. Los efectos adversos (p. ej., prurito, urticaria) se controlan relativamente bien con premedicación mediante antihistamínicos. Individuos con signos de EG tipo 2 y aquellos con hidrops fetalis no son candidatos apropiados para trasplante de médula ósea, TRE o TRS. Los pacientes con EG tipo 3 parecen obtener algún beneficio de la TRE, aunque el 
pronóstico a largo plazo aún está por definir para este grupo heterogéneo. El TRE parece reducir el riesgo de aborto espontáneo y las complicaciones durante el parto y el puerperio, sin presentar efectos sobre el feto ni niños lactantes ${ }^{9}$.

2. Terapia de reducción de sustrato(TRS).

Tiene como objetivo restaurar la homeostasis metabólica limitando la cantidad de precursor de sustrato sintetizado (y eventualmente sujeto a catabolismo); pudiendo ser procesado de manera eficaz por la enzima mutada con actividad hidrolítica residual. El Miglustat es el primer agente oral en pacientes con EG leve a moderada para quienes la TRE no es una opción terapéutica ${ }^{6}$. Eliglustat, inhibidor de la glucosilceramidasintetasa, ha demostrado mejoría o estabilización de las cifras de hemoglobina y plaquetas y de los volúmenes de bazo e hígado así como de los parámetros óseos en pacientes naive. Está aprobado a una dosis de 84 mg dos veces al día para metabolizadores rápidos o intermedios y de 84 mg una vez al día para metabolizadores lentos y para los pacientes que toman ciertos medicamentos metabolizados por la vía del citocromo P450 (CYP) 2D6. Los efectos secundarios de eliglustat fueron generalmente leves o moderados y transitorios, incluyendo dispepsia, cefalea, dolor abdominal, diarrea, nauseas, artralgias entre los mas frecuentes ${ }^{18}$.

Aunque la terapia de reemplazo enzimático (TRE) ha cambiado la historia natural de la EG, pueden requerir asociación de otro tipo de tratamientos como analgesia, terapia para la osteoporosis, cirugía de reemplazo articular, transfusión de productos sanguíneos, etc.

\section{BIBLIOGRAFÍA}

1. Nalysnyk L, Rotella P, Simeone JC, Hamed A, Weinreb N. Gaucher disease epidemiology and natural history: a comprehensive review of the literature. Hematology. 2017 Mar;22(2):65-73

2. Mistry PK, Belmatoug N, vom Dahl S, Giugliani R. Understanding the natural history of Gaucher disease. Am J Hematol. 2015 Jul;90 Suppl 1:S6-11

3. Koprivica V, Stone DL, Park JK, Callahan M, Frisch A, Cohen IJ, Tayebi N, Sidransky E. Analysis and classification of 304 mutant alleles in patients with type 1 and type 3 Gaucher disease. Am J Hum Genet. 2000 Jun;66(6):1777-86

4. Stirnemann J, Belmatoug N, Camou F, Serratrice C, Froissart R, Caillaud C, Levade T, Astudillo L, Serratrice J, Brassier A, Rose C, Billette de Villemeur T, Berger MG. A Review of Gaucher Disease Pathophysiology, Clinical Presentation and Treatments. Int J Mol Sci. 2017 Feb 17;18(2):44

5. Sidransky E. Gaucher disease: complexity in a "simple" disorder. Mol Genet Metab. 2004 Sep-0ct;83(1-2):6-15

6. Gary SE, Ryan E, Steward AM, Sidransky E. Recent advances in the diagnosis and management of Gaucher disease. Expert Rev Endocrinol Metab. 2018 Mar;13(2):107-118

7. Pastores GM, Weinreb $\mathrm{NJ}$, Aerts $\mathrm{H}$, et al. Therapeutic goals in the treatment of Gaucher disease. Semin Hematol. 2004 0ct;41:4-14

8. Hughes D, Mikosch P, Belmatoug N, Carubbi F, Cox T, Goker-Alpan 0, Kindmark A, Mistry P, Poll L, Weinreb N, Deegan P. Gaucher Disease in Bone: From Pathophysiology to Practice. J Bone Miner Res. 2019 Jun;34(6):996-1013

9. Zimran A, Morris E, Mengel E, Kaplan P, Belmatoug N, Hughes DA, Malinova V, Heitner R, Sobreira E, Mrsi区 M, Granovsky-Grisaru S, Amato D, vom Dahl S. The female Gaucher patient: the impact of enzyme replacement therapy around key reproductive events (menstruation, pregnancy and menopause). Blood Cells $\mathrm{Mol}$ Dis. 2009 Nov-Dec;43(3):264-88.

10. Charrow J, Andersson HC, Kaplan P, Kolodny EH, Mistry P, Pastores G, Rosenbloom BE, Scott CR, Wappner RS, Weinreb NJ, Zimran A. The Gaucher registry: demographics and disease characteristics of 1698 patients with Gaucher disease. Arch Intern Med. 2000 0ct 9;160(18):2835-43.

11. Pastores GM, Hughes DA. Gaucher Disease. 2000 Jul 27 [updated 2018 Jun 21]. In: Adam MP, Ardinger HH, Pagon RA, Wallace SE, Bean LJH, Stephens K, Amemiya A, editors. GeneReviews ${ }^{\circledR}$ [Internet]. Seattle (WA): University of Washington, Seattle; 1993-2020. PMID: 20301446./

12. Revel-Vilk S, Fuller M, Zimran A. Value of Glucosylsphingosine (Lyso-Gb1) as a Biomarker in Gaucher Disease: A Systematic Literature Review. Int J Mol Sci. 2020 Sep 28;21(19):7159

13. Razek AAKA, Abdalla A, Barakat T, El-Taher H, Ali K. Assessment of the liver and spleen in children with Gaucher disease type I with diffusion-weighted MR imaging. Blood Cells Mol Dis. 2018 Feb;68:139-142

14. Andrade-Campos M, Valero E, Roca M, Giraldo P; Spanish group on Gaucher Disease. The utility of magnetic resonance imaging for bone involvement in Gaucher disease. Assessing more than bone crises. Blood Cells Mol Dis. 2018 Feb;68:126-134.

15. Biegstraaten M, Cox TM, Belmatoug N, Berger MG, Collin-Histed T, Vom Dahl $S$, et al. Management goals for type 1 Gaucher disease: An expert consensus document from the European working group on Gaucher disease. Blood Cells Mol Dis. 2018 Feb;68:203-208

16. Ganz ML, Stern S, Ward A, Nalysnyk L, Selzer M, Hamed A, Weinreb N. A new framework for evaluating the health impacts of treatment for Gaucher disease type 1. Orphanet J Rare Dis. 2017 Feb 20;12(1):38

17. Revel-Vilk S, Szer J, Mehta A, Zimran A. How we manage Gaucher Disease in the era of choices. Br J Haematol. 2018 Aug;182(4):467-480

18. Belmatoug N, Di Rocco M, Fraga C, Giraldo P, Hughes D, Lukina E, MaisonBlanche P, Merkel M, Niederau C, PI凶ckinger U, Richter J, Stulnig TM, Vom Dahl S, Cox TM. Management and monitoring recommendations for the use of eliglustat in adults with type 1 Gaucher disease in Europe. Eur J Intern Med. 2017 Jan;37:25-32 